\title{
A Comparative Study of X ray Diffraction Pattern Obtaining Methods for Portable Diffractometer
}

\author{
Fang Gong1*, Xiaoyong $\mathrm{Ni}^{2}$, Dianhong Wang${ }^{2}$, Xingfeng $\mathrm{Guo}^{3}$ \\ ${ }^{1}$ School of Automation, China University of Geosciences, No. 388, Lumo Road, Wuhan, China, 430074 \\ 2 Department of Mechanical and Electronic information, China University of Geosciences, No. 388, Lumo \\ Road, Wuhan, China, 430074. \\ ${ }^{3}$ School of Electrical and Information Engineering, Wuhan Institute of Technology, No. 206, Guanggu Road, \\ Wuhan, , China, 430074.
}

*Corresponding author: Tel. 18062599165; email:18062599165@163.com

Manuscript submitted July 8, 2017; accepted September 5, 2017.

doi: 10.17706/jsw.12.12.914-922

\begin{abstract}
With the development of portable X-ray diffractometer for in-situ analysis, powder diffraction patterns are obtained in a diffractometer with a two-dimensional area detector. However, detector system and experimental geometry introduces distortion into acquired data. To obtain accurate diffraction pattern information, it is necessary to apply calibrations. Position of the X-ray incident beam centre on the detector and any non-orthogonality of the detector to the direct beam are the primary considerations. Although many calibration methods have been developed to calculate the intensity-scattering-angle dependence, there are few comparation between them. The purpose of this paper is to investigate these methods. The methods considered include the histogram method, the coordinate transformation method and the Nelder-Mead Simplex method. From the achievement of qualitative analysis angle, we experimentally compare the performance of the methods and illustrate properties such as accuracy and computational efficiency.
\end{abstract}

Keywords: X-ray diffraction pattern, calibration methods, computational efficiency, accuracy.

\section{Introduction}

Starting from the early 1990s, the first prototype of mobile x-ray diffraction (XRD) instrument was developed at NASA. A few commercial companies (e.g. CHEMIN \& MOXTEK) started to manufacture portable XRD instruments [1]-[3]. Even though a portable XRD instrument achieves the goal for remote in-situ mineralogical characterization of geosciencal materials marks contrast to traditional one, it sacrifices the accuracy of obtaining diffraction pattern. In order to obtain accurate intensity, position of the beam centre on the detector and any non-orthogonality of the detector to the direct beam need to be considered.

An interpolation method was developed at first to get a one-dimensional diffraction pattern from two-dimensional data [5]. Polar grids were set around the beam centre. The intensity at each grid point was interpolated from data at $9 \times 9$ pixels around the grid point. The intensity-angle plot was obtained by integrating the intensity along the azimuthal direction at each radial step. But this method was found to be valid when the half width of the diffraction peak was larger than five pixels. A histogram method was alternatively developed [6]. Intensity at each pixel was plotted as a function of the distance from the direct 
beam position. This plot was segment with an appropriate interval and the intensities in each segment were averaged. The histogram method is more flexible in integration and needs less computing time than the interpolation method. These algorithms are based on the ideal condition, the $\mathrm{X}$ ray incident beam is absolutely perpendicular to the detector plane.

The ideal alignment is very hard to achieve, therefore inaccuracies in the alignment are calculated and corrected during the post processing of the diffraction data. Calculation of the beam center defined over a fixed 2 range has been used [8]. This provides a simple method, but care is needed as non-zero backgrounds can lead to bias in the results. A much better method is cross-correlation with a known peak profile function [9]. The peak centre of each diffraction ring is estimated to be the position where the cross-correlation value is a maximum. This produces better estimates of position for weak and noisy data, and can overcome the problem of sloping backgrounds for symmetric peak profiles [4],[9]. Some authors proposed a algorithm based on the histogram method [7], which used a coordinates to partition the two-dimension data into sectors and the pixels within each sector were rebinned in terms of the distance in pixels from the point with these coordinates. This algorithm used varies the unknown parameters until the data rebinned are maximally sharp. When it reaches the maximally sharp, it achieves the calibration. This method seems appropriate for obtaining a number of coordinates for each diffraction ring. However, a criterion for determining the optimum number of sectors is lacking. With the extensively use of Optimization algorithm, the Nelder-Mead Simplex Method [10],[12] has been used. This algorithm works by giving an initial guess for the four parameters of the select function and then searches from this point to find any local minima in that function, within a selected tolerance. This method did not exploit any coordinates of experimental or diffraction geometry and was convenient. The only disadvantages of this method is it cannot find the correct minima without sharp peaks. Full Width at half maximum also be used to calibrate the diffraction pattern [11]. In this method, the position of the centre is refined by $\mathrm{N}$ pixels to the left and right. The array is radially integrated and the FWHM of the diffraction rings ia calculated. The beam position is then refined until the highest angular resolution is achieved.

Invariably these methods can obtain the $2 \theta$ diffraction plots ultimately while the conversion results are different from each other and research on comparisons between each other is sparse. We feel that it is necessary to put a number of these algorithms together and examine their properties in more detail. The study is experimental and the images data are obtained using Terra-503. Terra-503 is the first portable XRD/XRF analyzer, which can be used for in-situ analysis on the field [13]. We compare the test results of Terra-503 for these processing methods and the results can be used as reference for other tests and experiments with different detect equipment.

This paper is organized as follows.In the next section we introduce the basic experimental geometry. This is followed by a brief description of radial integration to convert diffraction images data into $2 \theta$ diffraction plots primarily. And then we introduce two mainly methods considered in our study. The experimental evaluation of each method is then given and the final section presents the conclusion of our work.

\section{Experimental Geometry}

Traditional X-ray diffractometers can easily obtain the intensity and diffraction angle data through the photon counting of photon detectors. It owns a high accuracy. However, since its huge size, these traditional X-ray diffractometers can only be used in laboratory. In order to achieve the goal of detecting in wild, various kinds of potable diffractometers are proposed, such as Terra-503 invented by CHEMIN. the breadboard set-up constructed in MOXTEK, and surface monitor acquired by the UGent Analytical Chemistry department.

We use the Terra-503 to obtain the XRD images in this paper. We can simplify X-ray diffraction structure 
to the following model (Fig. 1). The incident X-ray beam is perpendicular to the two-dimensional detector (e.g. charge couple device \& image plate). The sample is placed between the X-ray tube and the detector, along the direction of the incident X-ray. X-ray is irradiated on the powder sample to produce diffraction, and a Debye diffraction ring is formed on the two-dimensional detector. The diffraction rings are useful in retrieving information about certain aspects of the sample, but we cannot obtain direct information about its identification. A certain method have to performed on the diffraction images to get the information in a standard $2 \theta$ diffraction plot. The following three sections describe the specific methods used in this process.

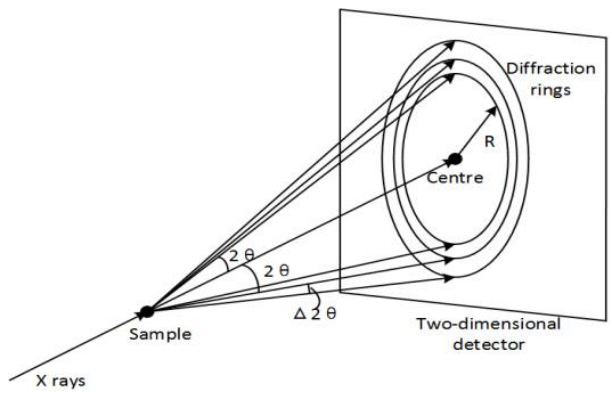

Fig. 1. Experimental geometry for portable X ray diffractometer Terra-503.

\section{Histogram Method}

A histogram method is developed in the integration as is illustrated in Fig. 2. Intensity at each pixel is plotted as a function of the distance from the direct beam position. The black triangle represents the intensity of each pixel. This plot describes a diffraction profile with random step. Then a curve fitting program is used for analyzing the diffraction data. The plot is segmented with an appropriate interval, and the intensities in each segment are averaged.

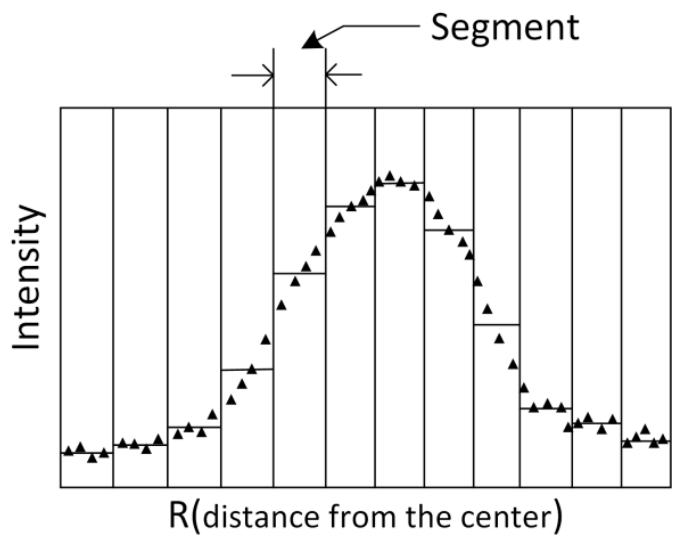

Fig. 2. Principles of the histogram method.

The process described above can be presented as:

$$
I_{\text {aver }}=\frac{\sum_{i=1}^{n} I\left(r_{0}+i \Delta r\right)}{\text { Photonnum }}
$$

where $I_{\text {aver }}$ is the intensities in each segment. $\mathrm{r}_{0}$ is a initial variable of radius. $\Delta \mathrm{r}$ is width of each segment along the direction of radius. Photonnum is the total number of photon at same radius. $n$ equals to the number of segments.

The histogram method is simple, assuming we know the location of the centre of the diffraction rings. For 
the simple integration it is also assumed that the collimated X-ray beam is at normal incidence to the detector. Unfortunately, this is not the case with the data we collect. The centre of the rings varies from sample to sample. The collimating optics of the detector has a natural tendency to move around slightly with time and adjustment. The centre of the rings needs to be found for each data set to get the correct $2 \theta$ plot. Base on histogram method, the next two section introduce methods to solve this problem.

\section{Coordinate Transformation Method}

The coordinate transformation method in this study mainly achieve the Beam center and detector tilt determination. For a tilted flat detector, it starts by considering a cone in the detector coordinate $\operatorname{system}\left(\mathrm{x}_{c}, \mathrm{y}_{c}, \mathrm{z}_{\mathrm{c}}\right)$. This is tilted, and rotated to the detector frame $\left(\mathrm{x}_{\mathrm{d}}, \mathrm{y}_{\mathrm{d}}, \mathrm{z}_{\mathrm{d}}\right)$. The cone is of opening angle $2 \theta$ and the apex is a distance $d$ from the sample along the $z_{c}$ axis. The cone is defined:

$$
x_{c}^{2}+y_{c}^{2}=\left(\left(d-z_{c}^{2}\right) \tan 2 \theta\right)^{2}
$$

The cone is tilted with respect to the perpendicular from the detector by an angle $\varphi$ in a plane. Eq.(2) transformed by an angle of $-\varphi$ about the yc axis to a coordinate frame $\left(\mathrm{x}_{\mathrm{t}}, \mathrm{y}_{\mathrm{t}}, \mathrm{z}_{\mathrm{t}}\right)$. For $\mathrm{z}_{\mathrm{t}}=0$ :

$$
\cos ^{2} \phi x_{t}^{2}+y_{t}^{2}=\left(d+\sin \phi x_{t}\right)^{2} \tan ^{2} 2 \theta
$$

The tilt plane has an angle of $\beta$ with respect to the $x_{c}$ axis of the detector frame. Eq.(3) transformed by an angle of $-\beta$ about the $z_{t}$ axis:

$$
\begin{aligned}
& \cos ^{2} \phi\left(\cos \beta x_{d}+\sin \beta y_{d}\right)^{2}+\left(-\sin \beta x_{d}+\cos \beta y_{d}\right)^{2} \\
& =\left(d+\sin \phi\left(\cos \beta x_{d}+\sin \beta y_{d}\right)\right)^{2} \tan ^{2} 2 \theta
\end{aligned}
$$

Eq.(4) is re-arranged to calculate an effective $2 \theta$ angle( $2 \theta^{\text {obs}}$ ) from a detector coordinate:

$$
2 \theta^{o b s}=\arctan \left(\frac{\cos ^{2} \phi\left(\cos \beta x_{d}+\sin \beta y_{d}\right)^{2}+\left(-\sin \beta x_{d}+\cos \beta y_{d}\right)^{2}}{\left(d+\sin \phi\left(\cos \beta x_{d}+\sin \beta y_{d}\right)\right)^{2}}\right)^{1 / 2}
$$

Here $x_{d}=x-X_{c}$ and $y d=y-Y_{c} .\left(X_{c}, Y_{c}\right)$ is the centre of the diffraction rings. Eq.(5) are used for non-linear least squares optimization of the beam centre and tilt. For $\mathrm{M}$ diffraction rings, each measured using $\mathrm{N}$ coordinates, the objective function to be minimized is :

$$
S=\sum_{j}^{M} \sum_{i}^{N}\left(\sqrt{I_{i j}}\left(2 \theta_{i j}^{o b s}-2 \theta_{j}^{\mathrm{mod} e l}\right)\right)^{2}
$$

where: $I_{i j}$ is the integrated profile intensity for the sector $\mathrm{i}$, on ring j; $2 \theta^{\text {obs }}$ is the calculated $2 \theta$ angle based on the ring coordinate and the current model value of the beam centre and tile; $2 \theta$ is the current model value of the $2 \theta$ angle of the ring $j$, see [6] for details.

\section{Nelder-Mead Simplex Method}

Nelder and Mead designed a new simplex search method based on the work of Spendley et al. The Nelder-Mead Simplex method attempts to minimize a scalar-valued nonlinear function $f(x)$ of $n$ real 
variables using only function values, without any derivative information. NMSM uses the operations of reflection $(\rho)$, expansion $(\chi)$, contraction $(Y)$ and shrinkage $(\sigma)$ to compare the target function values of $n+1$ vertices of the simplex, and then replaces the maximum vertices of the target function with new points. By constantly iteration and updating the simplex, the final simplex will be the optimal solution of the approximation problem. One iteration of the Nelder-Mead Simplex method can be described as:

1. Order. Order the $\mathrm{n}+1$ vertices to satisfy, $f\left(x_{1}\right) \leq f\left(x_{2}\right) \leq \cdots \leq f\left(x_{n+1}\right)$ using the tie-breaking rules given below.

2. Reflect. Compute the reflection point $\mathrm{x}_{\mathrm{r}}$ from

$$
x_{r}=\bar{x}+\rho\left(\bar{x}-x_{n+1}\right)
$$

where $\bar{x}=\sum_{i=1}^{n} x_{i} / n$ is the centroid of the $\mathrm{n}$ best points (all vertices except for $\mathrm{x}_{\mathrm{n}+1}$ ). Evaluate $f_{r}=f\left(x_{r}\right)$ If $f_{1}<f_{r}<f_{n}$, accept the reflected point $\mathrm{x}_{\mathrm{r}}$ and terminate the iteration.

3. Expand. If $f_{r}<f_{1}$,calculate the expansion point xe,

$$
x_{e}=\bar{x}+\chi\left(x_{r}-\bar{x}\right)
$$

And evaluate $f_{e}=f\left(x_{e}\right)$. If $f_{e}<f_{r}$, accept $\mathrm{x}_{\mathrm{e}}$ and terminate the iteration; otherwise, accept $\mathrm{x}_{\mathrm{r}}$ and terminate the iteration.

4. Contract. If $f_{r}>f_{n}$, perform a contraction betweens $\bar{x}$ and the better of $\mathrm{x}_{\mathrm{n}+1}$ and $\mathrm{x}_{\mathrm{r}}$.

a. Outside. If $f_{n} \leq f_{r} \leq f_{n+1}$, perform an outside contraction:

$$
x_{c}=\bar{x}+\gamma\left(x_{r}-\bar{x}\right)
$$

Evaluate $f_{c}=f\left(x_{c}\right)$. If $f_{c} \leq f_{r}$, accept $\mathrm{x}_{c}$ and terminate the iteration; otherwise, go to the step 5 .

b. Inside. If $f_{r} \geq f_{n+1}$, perform an inside contraction:

$$
x_{c c}=\bar{x}-\gamma\left(\bar{x}-x_{n+1}\right)
$$

Evaluate $f_{c c}=f\left(x_{c c}\right)$. If $f_{c c}<f_{n+1}$, accept $\mathrm{x}_{c c}$ and terminate the iteration; otherwise, go to the step 5 .

5. Shrink. Evaluate $f$ at the n points $v_{i}=x_{1}+\sigma\left(x_{i}-x_{1}\right), i=2, \ldots, n+1$. The vertices of the simplex at the next iteration consist of $x_{1}, v_{2}, \ldots, v_{n+1}$.

In order to automatically generate the diffraction pattern, a selected function is minimized. By minimizing the selected function, the beam centre and detector tilt angle are acquired. The minimzed function is the function that describes the area under a line and under the $2 \theta$ plot itself (Fig. 3). The function value is the area of the shaded region. This method chooses a line by multiply the total area under the $2 \theta$ plot curve by four or five. When the area in the peaks above the line is maximized, the area under the peaks is simultaneously minimized. The area under the line is minimized when the Nelder-Mead Simplex method has been performed correctly. This method works by giving a initial guess for the four parameters of the function. It then searches from this point to find any local minima in that function, within a selected tolerance. The four input variables include two numbers for the centre of the rings and two numbers for the 
angles that describe the detector tilt. Theory and application of the method can be found in [10],[12].

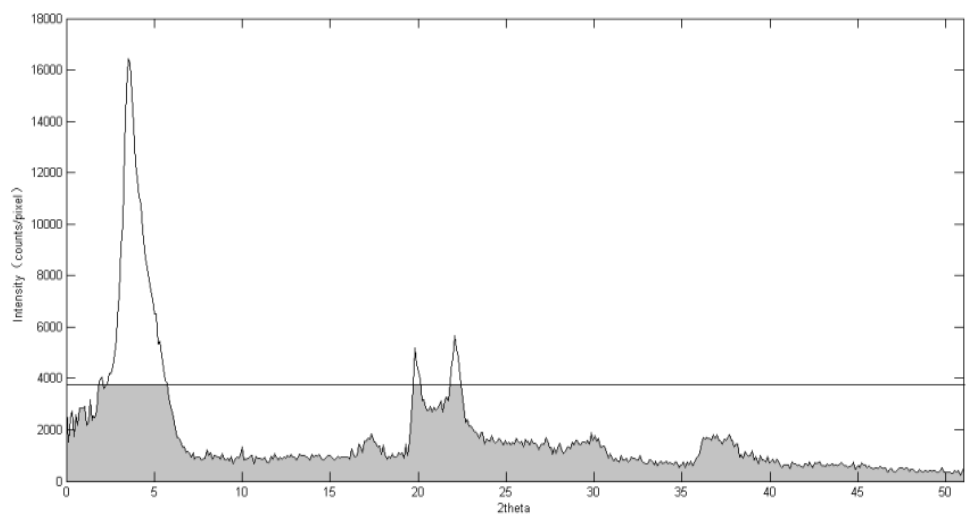

Fig. 3. Co K $\alpha 2 \theta$ diffraction plot (Bentonite sample). Histogram method perform knowing the center of the diffraction rings.

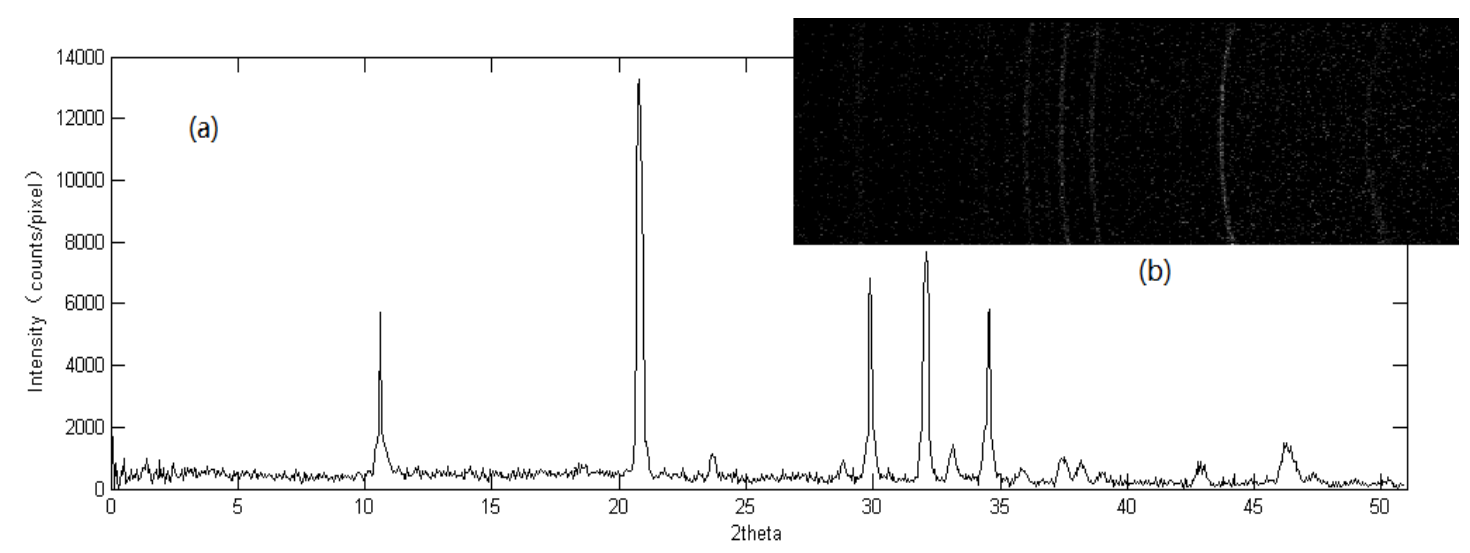

Fig. 4. (a) Co K $\alpha$ I-2 $\theta$ diffraction plot (gypsum sample). (b) XRD image detected by Terra-503.

\section{Experimental Comparison}

There are many criteria which can be considered in any comparison of algorithms, but in our study the most important points relate to the accuracy and computational efficiency. The accuracy is measured by comparing the gravity angle of each diffraction peaks, the beam centre and the detector tilt with geometry parameters of Terra-503. To test the calibration property of each methods, sample of Gypsum(CaSO4-2H20) is examined. The locations, error between the observed and the reference are calculated for each methods. To estimate computational efficiency we use the the time taken to run each integration algorithm on our ideapad $710 \mathrm{~S}$ computer. We realize that as measure of efficiency this is not necessarily of general significance. However at least in a coarse sense we would expect the conclusions regarding efficiency to hold. The major time consumption for each algorithm occurs for the centre location of the x-ray rings and it is easy to be caught in an endless loop if the accuracy is high. Note that all the algorithms were coded in MATLAB.

Fig. 4(a) shows the $2 \theta$ diffraction plot (gypsum sample) integrated by histogram method. We assume that the calibration parameters are under ideal circumstances: $\mathrm{Xc}=1024, \mathrm{Yc}=128$, tiltangle $=0^{\circ}$. Radial step is 2pixels $\left(\sim 0.099^{\circ}\right)$. Fig. 4 (b) shows a XRD image detected by Terra-503. 71 times exposure plus 179 times without $X$ ray irradiation detected by Terra-503. The range of the diffraction angle is $5-70^{\circ}$. In the geometry system of the Terra-503, the distance between powder sample and detector $(256 \times 1024)$ is $30 \mathrm{~mm}$. The experiment results illustrate that histogram method can readily obtain the XRD pattern transformation 
from a XRD image. There is a one-to-one correspondence between the diffraction peaks and rings. However, the intensities and $2 \theta$ value may not be accurate because of a deviation of beam centre location and detector tilt.

Due to the finite data points in the two-dimensional detector, we can only obtain parts of the whole diffraction rings(Fig. 4(b)). So, it is difficult for us to determine the beam centre and detector tilt manually. The coordinate transformation method and Nelder-Mead Simplex method were performed on the diffraction images to get the optimal $2 \theta$ diffraction plot. Fig. 5 shows the I- $2 \theta$ diffraction plot of $(020)$, crystal plane array index, line using three different methods. It is obviously that three methods have difference in performance. The blue plot shows a more wider and lower peak than other two plots because of the deviation of beam centre and detector tilt. The gravity of the red and green plots are more closer to the reference value $\left(2 \theta=11.70^{\circ}\right)$. The peaks of the the green plot is more sharper and a little bit higher than the red one. We can conclude that using the coordinate transformation method and Nelder-Mead Simplex method really achieve more accurate results than using only histogram method.

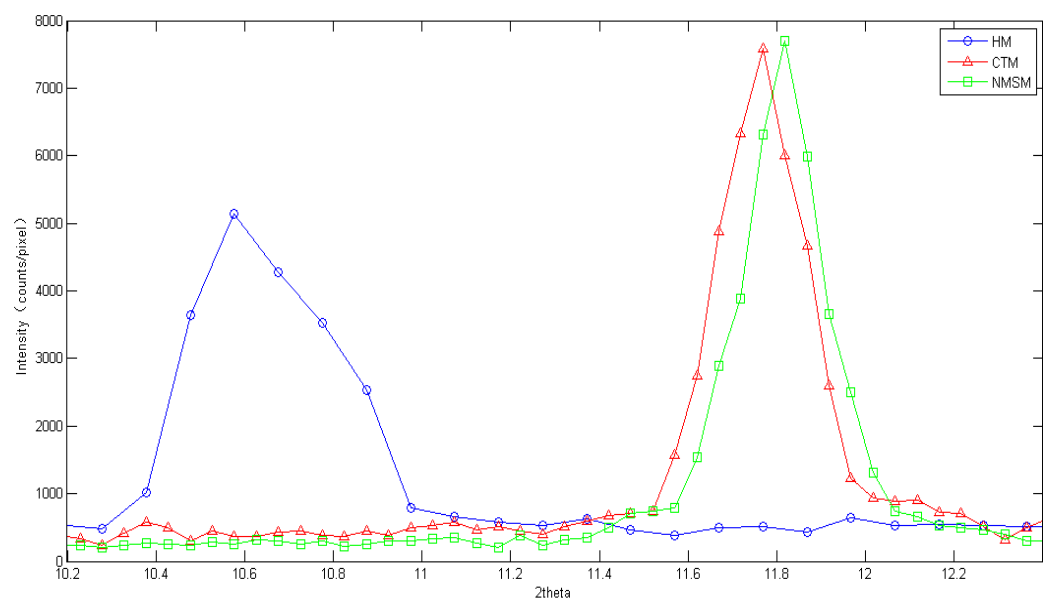

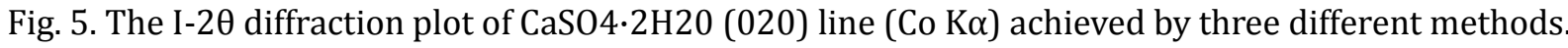

Table 1. The Reference and Observed Scattering Angle $(\lambda=1.7889 \AA)$

\begin{tabular}{cccccccc}
\hline \hline reflection & 020 & -121 & 031 & -141 & 002 & 022 & -123 \\
\hline $2 \theta_{\text {ref }}\left({ }^{\circ}\right)$ & 11.70 & 20.79 & 23.45 & 29.17 & 31.17 & 33.42 & 43.47 \\
$2 \theta_{\text {HMobs }}\left({ }^{\circ}\right)$ & 10.58 & 19.80 & 23.68 & 29.89 & 32.07 & 33.12 & 42.95 \\
$2 \theta_{\text {CTMobs }}\left({ }^{\circ}\right)$ & 11.77 & 20.88 & 23.35 & 29.18 & 31.10 & 33.43 & 43.55 \\
$2 \theta_{\text {MMSMobs }}\left({ }^{\circ}\right)$ & 11.80 & 20.81 & 23.44 & 29.27 & 31.09 & 33.50 & 43.49 \\
\hline \hline
\end{tabular}

Fig. 5 only show the first peak of the whole diffraction plot to illustrate the specific difference between the three methods. Table 1 lists the reflection, the reference angle and observed angle of each peaks. The wave length of the X-ray is $1.7889 \AA$. The average error obtained by the formula

$$
\sigma=\sum\left|2 \theta_{o b s}-2 \theta_{r e f}\right| / 7
$$

where $\sigma_{\mathrm{HM}}=0.6828, \sigma_{\mathrm{CTM}}=0.0585, \sigma_{\mathrm{NMSM}}=0.0641$. this results show that the Nelder-Mead Simplex method has a calibration capability similar to that of the coordinate transformation method, and sometimes even better.

In these methods, the integration time depend directly on the method complexity. The NMSM algorithm 
contains the local optimization process, which will spend a lot of time. Considering the integration time of each of the 3 algorithms, as shown in table 2 we see that the HM method has a clear advantage over the others. The CTM is second best. The integration time of the NMSM is rather unpredictable. It will depend on the iteration times and quantity (SNR) of the XRD images. Otherwise, it will be trapped into local optima.

Table 2. The Integration Time Requirement for Each Algorithm

\begin{tabular}{cccc}
\hline \hline Method & HM & CTM & NMSM \\
\hline Integration Time(s) & 9.755 & 118.746 & 1273.251 \\
\hline \hline
\end{tabular}

\section{Conclusion}

The results of our study indicate that the HM can obtain the $2 \theta$ plot converting from XRD images easily if the diffraction geometry in a ideal circumstances. However, the ideal alignment is very difficult to achieve. So, the results always deviate from standard reference position. The CTM and NMSM can achieve the determination of beam centre and detector tilt based on the HM. The CTM is the most widely used algorithm. Although its calibration accuracy is close to the NMSM, its integration time is far less than the NMSM, which means it can be applied to the situation that requires a higher accuracy and efficiency. The NMSM suffers from the common problem of the most optimization algorithms, that is, their results can easily fall into local optimum. We have found, the NMSM cannot find the correct minima without obvious diffraction rings. However, it can be solved by having a post processing of the diffraction data. Based on the above researches, our work of next step is studying the machine learning algorithms and training a unified model, which take the advantages of high precision and overcome the low efficiency of the NMSM.

\section{Acknowledgement}

The work was partially supported by the Natural Science Foundation of Hubei Province (2014BEC073).

\section{References}

[1] Voorde, L. V. D., Vekemans, B., Verhaeven, E., Tack, P., \& Wolf, R. D. (2015). Analytical characterization of a new mobile $\mathrm{x}$-ray fluorescence and x-ray diffraction instrument combined with a pigment identification case study. Spectrochimica Acta Part B Atomic Spectroscopy, 110, 14-19.

[2] Vaniman, D., Bish, D., Guthrie, G., Chipera, S., Blake, D., Collins, S. A., Elliott, S. T., \& Sarrazin, P. (1999). Processing monitoring and control with CHEMIN, a miniaturized CCD-based instrument for simultaneous XRD/XRF analysis. Proceedings of the SPIE 3679.

[3] Sarrazin, P., Blake, D., Feldman, S., Chipera, S., Vaniman, D., \& Bish, D. (2004). Field deployment of a portable XRD/XRF instrument on mars analog terrain. Advances in X-Ray Analysis.

[4] Hammersley, A. P., Svensson, S. O., \& Thompson, A., et al. (1995). Calibration and correction of distortions in two-dimensional detector systems. Review of Scientific Instruments, 66(2), 2310-2310.

[5] Shimomura, 0., Takemura, K., Fujihisa, H. et al. (1992). Application of an immaging plate to high-pressure x ray study with a diamond anvil cell. Review of Scientific Instruments, 63(1), 967-973.

[6] Sulyanov, S. N., Popov, A. N., \& Kheiker, D. M. (1994). Using a two-dimentional detector for x-ray powder diffractometry. Journal of Applied Crystallography, 27(6), 934-942.

[7] Piltz, R. O., McMahon, M. I., Crain, J., Hatton, P. D., Nelmes, R. J., Cernik, R. J., \& Bushnell-Wye, G. (1992). Review of Scientific Instruments, 63(1), 700-703.

[8] Meade, C., \& Jeanloz, R. (1990). High precision powder X-ray diffraction measurements at high pressures. Review of Scientific Instruments, 61(1), 2571-2580.

[9] Thomas, D. J. (1986). The development of a full profile analysis of single-crystal $x$ ray diffraction data. 
Le Journal De Physique Colloques.

[10] Lagarias, L., Reeds, J., Wright, P., \& Wright, M. (1998). Convergence properties of the nelder - mead simplex method in low dimensions. Saim Journal on Optimization a Publication of Society for Industrial \& Applied Mathematics. Optim, 9(1), pp. 112-147.

[11] Insitar, A. (2010). A portable CCD array detector for in-situ analysis of powder samples using combined.

[12] Cornaby, S., Reyesmena, A., Pew, H. K., Moody, P. W., Hughes, T., Stradling, A., \& Knight, L. V. (2001). Using a charged-coupled device (CCD) as an x-ray single photon energy-dispersive detector. Journal of $\mathrm{X}$-ray science and technology, 9(2), 85-97.

[13] Bish, D. L., Blake, D., Sarrazin, P., Treiman, A., \& Hoehler, T. (2007). Field XRD/XRF mineral analysis by the MSL CheMin instrument. Experimental \& Translational Stroke Medicine, 5(1), 12.

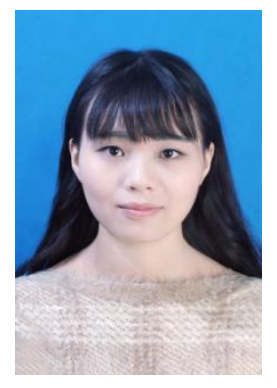

Fang Gong was born in Sichuan province, China, in 1991. She graduated in electronic information engineering from China University of Geosciences in 2014. She obtained a master's degree in information and communications engineering from China University of Geosciences in 2016. She is a Ph.D student at Faculty of Automation, China University of Geosciences from August 2016 until the present time. Her current scientific research primarily focuses on data mining and machine learning.

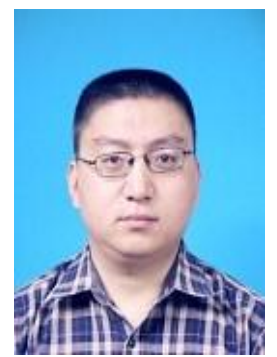

Xiaoyong $\mathbf{N i}$ is an associate professor at the Department of Electron Information Engineering, China University of Geosciences. He received his Ph.D in earth exploration technology from China University of Geosciences in 2009. His current scientific research primarily focuses on path planning and intelligence control algorithms.

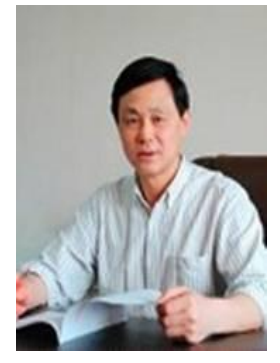

Dianhong Wang is a professor at the Department of Electron Information Engineering, China University of Geosciences. He received his Ph.D in pattern recognition and intelligence system from Huazhong University of Science and Technology in 2002. His current scientific research primarily focuses on intelligence instrument and computer application.

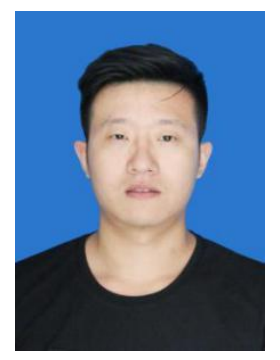

Xingfeng Guo is an instructor at the Department of Electrical and Information, Wuhan University of Institute and Technology. He received his Ph.D in earth exploration technology from China University of Geosciences in 2015. His current scientific research primarily focuses on image processing and WSN. 